\title{
ON THE INVERSE PROBLEM FOR STURM-LIOUVILLE OPERATOR WITH A NONLINEAR SPECTRAL PARAMETER IN THE BOUNDARY CONDITION
}

\author{
KhanlaR R. MAMEdov
}

\begin{abstract}
The inverse scattering problem is investigated for some second order differential equation with a nonlinear spectral parameter in the boundary condition on the half line $[0, \infty)$. In the present paper the coefficient of spectral parameter is not a pure imaginary number and the boundary value problem is not selfadjoint. We define the scattering data of the problem, derive the main integral equation and show that the potential is uniquely recovered.
\end{abstract}

\section{Introduction}

We consider the inverse problem of scattering theory on the half line $[0, \infty)$ for the equation

$$
-y^{\prime \prime}+q(x) y=\lambda^{2} y
$$

with the boundary condition

$$
y^{\prime}(0)-\left(\alpha_{0}+\alpha_{1} \lambda\right) y(0)=0,
$$

where $\lambda$ is spectral parameter, the potential $q(x)$ is real valued and satisfying the condition

$$
\int_{0}^{\infty}(1+x)|q(x)| d x<\infty
$$

$\alpha_{0}, \alpha_{1}$ is real numbers, also $\alpha_{0}, \alpha_{1} \geq 0$. The inverse problem of scattering theory on the half line for the boundary problem value (1.1)-(1.3) in the case $\alpha_{1}=0$ was completely solved in $[1,2,4,5,10,11]$. Inverse problems in the half line with the spectral parameter contained in the boundary conditions was investigated according to spectral function in [13], according to Weyl function in $[14,15]$, according to scattering data in [7]-[9]. Some of the applications of these

Received April 8, 2008.

2000 Mathematics Subject Classification. Primary 34B07, 34A55, 34B24.

Key words and phrases. inverse problem of scattering theory on half line, Sturm-Liouville operator with a nonlinear spectral parameter, scattering data.

This research is supported by the Scientific and Technical Research Council of Turkey (TUBITAK NATO PC-BC). 
problems to wave equation were given in [14]. Spectral analysis involving linear dependence on the spectral parameter in the boundary condition was studied in [3]. In this paper we prove the uniqueness of a solution of the inverse problem of scattering theory for the boundary problem (1.1)-(1.3) on the half line. As different from previous works $[7,8]$, in the present paper the coefficient $\lambda$ in boundary condition is not a pure imaginary number. Therefore, the boundary value problem (1.1)-(1.3) is not selfadjoint and it may have complex eigenvalues (see [6] and [12], Appendix II).

The paper is organized as follows. In Section 2 the scattering data for boundary value problem (1.1)-(1.3) is defined and some of their properties are examined. The main equation, which relate the scattering data, are derived in Section 3. In Section 4, we prove the uniqueness of the solution of the inverse problem.

It is known [11] that for any $\lambda$ from the closed upper half plane, equation (1.1) has a unique solution $e(x, \lambda)$ that can be represented in the form

$$
e(x, \lambda)=e^{i \lambda x}+\int_{x}^{\infty} K(x, t) e^{i \lambda t} d t
$$

and the kernel $K(x, t)$ satisfies the inequality

$$
\begin{gathered}
|K(x, t)| \leq \frac{1}{2} \sigma\left(\frac{x+t}{2}\right) \exp \left\{\sigma_{1}(x)-\sigma_{1}\left(\frac{x+t}{2}\right)\right\} \\
K(x, x)=\frac{1}{2} \int_{x}^{\infty} q(t) d t
\end{gathered}
$$

where

$$
\sigma(x) \equiv \int_{x}^{\infty}|q(t)| d t, \quad \sigma_{1}(x) \equiv \int_{x}^{\infty} \sigma(t) d t .
$$

The solution $e(x, \lambda)$ is an analytic function of $\lambda$ in the upper half plane $\operatorname{Im} \lambda \geq 0$ and is continuous on the real line. Moreover, the function $e(x, \lambda)$ posses the following properties:

$$
\begin{aligned}
|e(\lambda, x)| & \leq \exp \left\{-\operatorname{Im} \lambda x+\sigma_{1}(x)\right\}, \\
\left|e(\lambda, x)-e^{i \lambda x}\right| & \leq\left\{\sigma_{1}(x)-\sigma_{1}\left(x+\frac{1}{|\lambda|}\right)\right\} \exp \left\{-\operatorname{Im} \lambda x+\sigma_{1}(x)\right\}, \\
\left|e^{\prime}(\lambda, x)-i \lambda e^{i \lambda x}\right| & \leq \sigma(x) \exp \left\{-\operatorname{Im} \lambda x+\sigma_{1}(x)\right\} .
\end{aligned}
$$

For real $\lambda \neq 0$ the functions $e(x, \lambda)$ and $e(x,-\lambda)$ form a fundamental solutions of equation (1.1) and their Wronskian is equal to $2 i \lambda$ :

$$
W\{e(x, \lambda), e(x,-\lambda)\}=e^{\prime}(x, \lambda,) e(x,-\lambda)-e(x, \lambda) e^{\prime}(x,-\lambda)=2 i \lambda .
$$

Let $\omega(x, \lambda)$ be a solution of the equation (1.1) satisfying the following initialvalue conditions

$$
\omega^{\prime}(0, \lambda)=\alpha_{0}+\alpha_{1} \lambda, \quad \omega(0, \lambda)=1 .
$$




\section{Scattering data}

Lemma 2.1. For any real number $\lambda \neq 0$, the following identity is valid

$$
\frac{2 i \lambda \omega(x, \lambda)}{e^{\prime}(0, \lambda)-\left(\alpha_{0}+\alpha_{1} \lambda\right) e(0, \lambda)}=e(x,-\lambda)-S(\lambda) e(x, \lambda),
$$

where

$$
S(\lambda)=\frac{e^{\prime}(0,-\lambda)-\left(\alpha_{0}+\alpha_{1} \lambda\right) e(0,-\lambda)}{e^{\prime}(0, \lambda)-\left(\alpha_{0}+\alpha_{1} \lambda\right) e(0, \lambda)}
$$

and

$$
S(\lambda)=\overline{S(-\lambda)}=[S(-\lambda)]^{-1} .
$$

Proof. Since the two functions $e(x, \lambda)$ and $e(x,-\lambda)$ form a fundamental system of solutions to equation (1.1) for all real $\lambda \neq 0$, we can write

$$
\omega(x, \lambda)=A^{-} e(x,-\lambda)+A^{+} e(x, \lambda) .
$$

The Wronskian of solutions of equation (1.1) does not depend upon $x$, then we get

$$
\begin{aligned}
W\{\omega(x, \lambda), e(x, \pm \lambda)\} & =\omega^{\prime}(0, \lambda,) e(0, \pm \lambda)-\omega(0, \lambda) e^{\prime}(0, \pm \lambda) \\
& =-e^{\prime}(0, \pm \lambda)+\left(\alpha_{0}+\alpha_{1} \lambda\right) e(0, \pm \lambda) .
\end{aligned}
$$

On other hand

$$
W\{\omega(x, \lambda), e(x, \pm \lambda)\}=\mp 2 i \lambda A^{\mp}
$$

Thus, we obtain

$$
\begin{aligned}
\omega(x, \lambda)= & \frac{1}{2 i \lambda}\left\{e^{\prime}(0, \lambda)-\left(\alpha_{0}+\alpha_{1} \lambda\right) e(0, \lambda)\right\} e(x,-\lambda) \\
& -\frac{1}{2 i \lambda}\left\{e^{\prime}(0,-\lambda)-\left(\alpha_{0}+\alpha_{1} \lambda\right) e(0,-\lambda)\right\} e(x, \lambda) .
\end{aligned}
$$

Since $q(x)$ is real, it follows that $e(0,-\lambda)=\overline{e(0, \lambda)}$, and hence that $\varphi(\lambda) \equiv$ $e^{\prime}(0, \lambda)-\left(\alpha_{0}+\alpha_{1} \lambda\right) e(0, \lambda) \neq 0$ for all real $\lambda \neq 0$. From the last equality we can obtain the equalities (2.1), (2.2), and (2.3). The lemma is proved.

Lemma 2.2. The function $\varphi(\lambda)$ may have only a finite number of zeros in the half plane $\operatorname{Im} \lambda>0$ and all these zeros don't lie on the imaginary axis. Moreover, the function $\lambda[\varphi(\lambda)]^{-1}$ is bounded in a neighborhood point $\lambda=0$.

Proof. Since $\varphi(\lambda) \neq 0$ for all real $\lambda \neq 0$, the point $\lambda=0$ is possible real zero of the function $\varphi(\lambda)$. It follows from the analyticity of the function $\varphi(\lambda)$ on upper half plane zeros of $\varphi(\lambda)$ form at most countable set. It is obtained from properties (1.7)-(1.9) of the function $e(x, \lambda)$ that the zeros of the function $\varphi(\lambda)$ form bounded set in half plane $\operatorname{Im} \lambda>0$. Therefore, the zeros of $\varphi(\lambda)$ form at most countable and bounded set having $\lambda=0$ as the only possible limit point.

Now let us show that the function $\varphi(\lambda)$ may have a finite number zeros in half plane $\operatorname{Im} \lambda>0$. Assume the converse. Suppose that $\varphi(\lambda)$ have a infinite 
number zeros $\mu_{n}, n=1,2, \ldots$. Then, the function $y_{n}=y\left(x, \mu_{n}\right)$ satisfies the equation

$$
-y_{n}^{\prime \prime}+q(x) y_{n}=\mu_{n}^{2} y_{n}
$$

and the boundary condition

$$
y_{n}^{\prime}(0)-\left(\alpha_{0}+\alpha_{1} \mu_{n}\right) y_{n}(0)=0 .
$$

Let's multiply both sides of the equation (2.4) by $\overline{y_{n}}$ and integrate this equation over $\lambda$ from 0 to $\infty$. Using (2.5) from which we further obtain, via an integration by parts,

$$
\mu_{n}^{2}-\alpha_{1}\left|y_{n}(0)\right|^{2} \mu_{n}-\alpha_{0}\left|y_{n}(0)\right|^{2}-\left(L y_{n}, y_{n}\right)=0
$$

where

$$
\left(L y_{n}, y_{n}\right)=\int_{0}^{\infty}\left(\left|y_{n}^{\prime}\right|^{2}+q(x)\left|y_{n}\right|^{2}\right) d x
$$

Then we obtain

$$
\mu_{n}=\frac{\alpha_{1}\left|y_{n}(0)\right|^{2} \pm \sqrt{\alpha_{1}^{2}\left|y_{n}(0)\right|^{4}+4 \alpha_{0}\left|y_{n}(0)\right|^{2}+4\left(L y_{n}, y_{n}\right)}}{2}
$$

Since $\mu_{n}$ is not a real number and $\alpha_{0} \geq 0$, then $\left(L y_{n}, y_{n}\right)<0$. From the inequalities $\left(L y_{1}, y_{1}\right)<0$ and $\left(L y_{2}, y_{2}\right)<0$, we obtain $\left(L\left(a y_{1}+b y_{2}\right), a y_{1}+b y_{2}\right)<0$ holds for arbitrary complex numbers $a, b$. Thus,

$$
\left(L\left(a_{i} y_{i}+b_{i} y_{i+1}\right), a_{i} y_{i}+b_{i} y_{i+1}\right)<0
$$

for arbitrary numbers $a_{i}, b_{i}(i=1,2, \ldots)$.

From the asymptotic formulas ([12, p. 445])

$$
y_{n}(x)=e^{i \lambda_{n} x}[1+o(1)],(x \rightarrow \infty)
$$

it follows that the function $y_{n}(x)$ are linear independent and $y_{n}(0) \neq 0$ for arbitrary number $n$.

Now, we construct the following series of functions

$$
z_{j}(x)=a_{j} y_{j}(x)+b_{j} y_{j+1}(x), \quad j=1,2, \ldots
$$

Here, $a_{j}$ and $b_{j}$ are unknown complex numbers yet. Clearly, the numbers $a_{j}$ and $b_{j}$ can be chosen such that the condition $z_{j}(0)=0$ holds. In this case, we shall show that the functions $z_{j}(x)$ are linear independent. In fact, from the equality

$$
c_{1} z_{1}(x)+c_{2} z_{2}(x)+\cdots+c_{n} z_{n}(x)=0,
$$

we write

$$
c_{1} a_{1} y_{1}+\left(c_{1} b_{1}+c_{2} a_{2}\right) y_{2}+\cdots+\left(c_{n-1} b_{n-1}+c_{n} a_{n}\right) y_{n}=0 .
$$

Since the functions $y_{n}$ are linear independent, we have

$$
\begin{aligned}
c_{1} a_{1} & =0, \\
c_{1} b_{1}+c_{2} a_{2} & =0, \\
& \ldots
\end{aligned}
$$




$$
c_{n-1} b_{n-1}+c_{n} a_{n}=0
$$

or $c_{1}=c_{2}=\cdots=c_{n}=0$.

Thus, the relations

$$
\begin{gathered}
-z_{n}^{\prime \prime}+q(x) z_{n}=\lambda_{n} z_{n} \\
z_{n}(0)=0
\end{gathered}
$$

hold.

Denote by the operator $L_{0}$ in the space $L_{2}(0, \infty)$ acting as $L_{0} y=-y^{\prime \prime}+q(x) y$ on the domain

$$
D\left(L_{0}\right) \equiv\left\{y(x) \mid y^{\prime}(x) \in A C[0, \infty),-y^{\prime \prime}+q(x) y \in L_{2}(0, \infty), y(0)=0\right\} .
$$

It follows from the relations $(2.6),(2.7)$ that $z_{n}(x) \in D\left(L_{0}\right)$ and $\left(L_{0} z_{n}, z_{n}\right)<0$. Hence, we obtain that the operator $L_{0}$ has only a infinite number negative eigenvalues. But this is impossible due to the condition (1.3) on $q(x)$ (see [11]). We obtain a contradiction. It follows that the function $\varphi(\lambda)$ may have only a finite number of zeros in upper half plane $\operatorname{Im} \lambda>0$.

Now let us show that the zeros of the function $\varphi(\lambda)$ are not pure imaginary number. Assume the converse, let $\varphi\left(\lambda_{0}\right)=0, \lambda_{0}=i \mu, \mu>0$. Then we obtain

$$
-i \alpha_{1}|y(0)|^{2} \mu=\mu^{2}+\alpha_{0}|y(0)|^{2}+(L y, y) .
$$

Since $\alpha_{0}, \alpha_{1}, \mu,|y(0)|^{2}$ and $(L y, y)$ are real values, the last equality gives an contradiction, i.e., $\lambda_{0}$ is not a pure imaginary number.

Entirely analogously to Lemma 3.1 .3 in [11] it is proved that the function $\lambda[\varphi(\lambda)]^{-1}$ is bounded on the neighborhood of zero. Lemma 2.2 is proved.

Lemma 2.3. The zeros of the functions $\varphi(\lambda) \equiv e^{\prime}(0, \lambda)-\left(\alpha_{0}+\alpha_{1} \lambda\right) e(0, \lambda)$ and $\varphi_{1}(\lambda) \equiv e^{\prime}(0,-\lambda)-\left(\alpha_{0}+\alpha_{1} \lambda\right) e(0,-\lambda)$ are complex conjugate each other and the number of these zeros is equal.

Proof. According to Lemma 2.2 the function $\varphi(\lambda)$ in upper half plane $\operatorname{Im} \lambda>0$ has finitely many zeros $\lambda_{k}, k=1,2, \ldots, n$ and $\overline{\varphi\left(\lambda_{k}\right)}=0$. From the properties $\overline{e\left(0, \lambda_{k}\right)}=e\left(0,-\bar{\lambda}_{k}\right), \overline{e^{\prime}\left(0, \lambda_{k}\right)}=e^{\prime}\left(0,-\bar{\lambda}_{k}\right)$ of the $e(x, \lambda)$ we have $\overline{\varphi\left(\lambda_{k}\right)}=$ $e^{\prime}\left(0,-\bar{\lambda}_{k}\right)-\left(\alpha_{0}+\alpha_{1} \bar{\lambda}_{k}\right) e\left(0,-\bar{\lambda}_{k}\right)=\varphi_{1}\left(\bar{\lambda}_{k}\right)$ or $\varphi_{1}\left(\bar{\lambda}_{k}\right)=0, k=1,2, \ldots, n$. That is zeros of the functions $\varphi(\lambda)$ and $\varphi_{1}(\lambda)$ are complex conjugate, and also the number of these zeros is equal. Lemma 2.3 is proved.

According to [6] the function $\varphi(\lambda)$ is called the denominator of the boundary value problem (1.1)-(1.3). Since the function $e(0, \lambda)$ is analytic in the upper half plane $\operatorname{Im} \lambda>0$. It is clear from the formulas (1.7)-(1.9) that the function $\varphi(\lambda)$ is analytic in upper half plane and the asymptotic formula

$$
\varphi(\lambda)=\alpha_{1}-i+O\left(\frac{1}{\lambda}\right)
$$

holds as $|\lambda| \rightarrow \infty$. 
By the singular values of the boundary value problem (1.1)-(1.3) we mean the roots of the equation $\varphi(\lambda)=0$ which satisfy the conditions $\lambda_{k} \neq 0, \operatorname{Im} \lambda_{k} \geq$ 0 (see [12], p. 306). According to Lemma 2.2 the set of singular values of the boundary value problem (1.1)-(1.3) is finite and non pure imaginary. The multiplicity $m_{k}$ of a root $\lambda_{k}$ of the equation $\varphi(\lambda)=0$ is called the multiplicity of the singular value $\lambda_{k}, k=1,2, \ldots, n$.

From the relation (2.2) and (2.8), for $\operatorname{Im} \lambda \geq 0$ and $|\lambda| \rightarrow \infty$, we get

$$
S(\lambda)=S_{0}+O\left(\frac{1}{\lambda}\right)
$$

where $S_{0}=\frac{\alpha_{1}+i}{\alpha_{1}-i}$. The function $S(\lambda)$ defined by the formula (2.2) is called the scattering function of the boundary value problem (1.1)-(1.3).

Lemma 2.4. The function $S_{0}-S(\lambda)$ is the Fourier transform of a function $F_{S}(x)$ of the form

$$
F_{S}(x)=F_{S}^{(1)}(x)+F_{S}^{(2)}(x),
$$

where $F_{S}^{(1)}(x) \in L_{1}(-\infty, \infty)$, whereas $F_{S}^{(2)}(x) \in L_{2}(-\infty, \infty)$ and

$$
\sup _{-\infty<x<\infty}\left|F_{S}^{(2)}(x)\right|<\infty \text {. }
$$

Proof. From the formula (1.4) it follows that

Denoting

$$
\begin{gathered}
e(\lambda, 0)=1+\int_{0}^{\infty} K(0, t) e^{i \lambda t} d t \\
e^{\prime}(\lambda, 0)=i \lambda-K(0,0)+\int_{0}^{\infty} K_{x}(0, t) e^{i \lambda t} d t .
\end{gathered}
$$

$$
\begin{gathered}
q_{0}=K(0,0), \quad \sigma_{0}(\lambda)=\lambda\left(i-\alpha_{1}\right), \\
K_{1}(t)=K_{x}(0, t)-\alpha_{0} K(0, t), \quad K_{2}(t)=\alpha_{1} K(0, t)
\end{gathered}
$$

and

for simplicity, we see that

$$
\widetilde{K}_{j}(-\lambda)=\int_{0}^{\infty} K_{j}(t) e^{i \lambda t} d t, \quad j=1,2
$$

$S_{0}-S(\lambda)=\frac{\left(1-S_{0}\right)\left(\alpha_{0}+q_{0}\right)+S_{0}\left[\widetilde{K}_{1}(-\lambda)-\lambda \widetilde{K}_{2}(-\lambda)\right]+\widetilde{K}_{1}(\lambda)-\lambda \widetilde{K}_{2}(\lambda)}{\sigma_{0}(\lambda)-\left(\alpha_{0}+q_{0}\right)+\widetilde{K}_{1}(-\lambda)-\lambda \widetilde{K}_{2}(-\lambda)}$.

Every one of the functions

$$
\tilde{f}_{1}(\lambda)=\frac{\alpha_{0}+q_{0}}{\sigma_{0}(\lambda)}, \quad \tilde{f}^{ \pm}(\lambda)=\frac{\widetilde{K}_{1}( \pm \lambda)-\lambda \widetilde{K}_{2}( \pm \lambda)}{\sigma_{0}(\lambda)},
$$

is the Fourier transformation of a summable function. Hence we have

$$
S_{0}-S(\lambda)=\frac{\widetilde{f}(\lambda)}{1+\widetilde{K}(-\lambda)}
$$


where

$$
\begin{aligned}
\widetilde{f}(\lambda) & =\left(1-S_{0}\right) \widetilde{f}_{1}(\lambda)+S_{0} \tilde{f}^{-}(\lambda)+\tilde{f}^{+}(\lambda), \\
\widetilde{K}(-\lambda) & =\widetilde{f}_{1}(\lambda)+\widetilde{f}^{-}(\lambda) .
\end{aligned}
$$

Now let us rewrite the last equality as

$$
\begin{aligned}
& S_{0}-S(\lambda)=\tilde{f}(\lambda)\left[\left\{1+\left(1-\widetilde{h}\left(\lambda N^{-1}\right) \widetilde{K}(-\lambda)\right\}^{-1}-1\right]\right. \\
& +\widetilde{f}(\lambda)-\widetilde{f}(\lambda)\left\{\frac{1}{1+\left\{1-\widetilde{h}\left(\lambda N^{-1}\right) \widetilde{K}(-\lambda)\right\}}-\frac{1}{1+\widetilde{K}(-\lambda)}\right\},
\end{aligned}
$$

where

$$
\widetilde{h}(\lambda)=\left\{\begin{array}{cl}
1, & \text { if }|\lambda|<1 \\
2-|\lambda|, & \text { if } 1 \leq \lambda \leq 2 \\
0, & \text { if }|\lambda|>2
\end{array}\right.
$$

is the Fourier transform of the function $h(x) \in L_{1}(-\infty, \infty)$. Also, $h\left(\lambda N^{-1}\right)$ is the Fourier transform of the function $h_{N}(x)=N h(x N)$, and

$$
\lim _{N \rightarrow \infty}\left\|f(x)-h_{N} * f(x)\right\|_{L_{1}}=0
$$

for all $f(x) \in L_{1}(-\infty, \infty)$, where $h_{N} * f(x)$ is the convolution of functions $h_{N}(x)$ and $f(x)$ from $L_{1}(-\infty, \infty)$. The convolution $h_{N} * f(x)$ of functions $h_{N}(x)$ and $f(x)$ from $L_{1}(-\infty, \infty)$ is defined as $h_{N} * f(x)=\int_{-\infty}^{\infty} h_{N}(x-t) f(t) d t$. If $\|f\|_{L_{1}}<1$, then the series (see [11, p. 190])

$$
-f(x)+f * f(x)-f * f * f(x)+\cdots
$$

converges in the metric of $L_{1}(-\infty, \infty)$, its sum belongs to this space and its Fourier transform is equal to

$$
-\tilde{f}(\lambda)+\left\{\tilde{f}(\lambda\}^{2}-\left\{\tilde{f}(\lambda\}^{3}+\cdots=\left\{1+\tilde{f}(\lambda\}^{-1}-1 .\right.\right.\right.
$$

We conclude, from (2.11) and the previous argument, that for $N$ large enough, the function $\left[1+\left\{1-\widetilde{h}\left(\lambda N^{-1}\right)\right\} \widetilde{f}(-\lambda)\right]^{-1}-1$ is the Fourier transform of function from $L_{1}(-\infty, \infty)$. It follows that the sum of the first two terms in the righthand side of (2.10) is also the Fourier transform of a summable function $F_{S}^{(1)}(x)$. Finally, since $\widetilde{h}\left(\lambda N^{-1}\right)=0$ for $|\lambda|>2 N$, the third term in the same formula vanishes for $|\lambda|>2 N$ and is bounded. As such, it is the Fourier transform of a bounded function $F_{S}^{(2)}(x) \in L_{2}(-\infty, \infty)$, and Lemma 2.4 is proved.

It is known (see [12, p. 299]) that, the equation (1.1) has a solution $\hat{e}(x, \lambda)$ which for every $\alpha>0$ and $\delta>0$, as $x \rightarrow \infty$

$$
\hat{e}(x, \lambda)=e^{-i \lambda x}[1+o(1)],(x \rightarrow \infty)
$$


uniformly in the domain $\operatorname{Im} \lambda \geq \alpha,|\lambda| \geq \delta$. The solution $\hat{e}(x, \lambda)$ is a holomorphic function of $\lambda$ in the $\operatorname{Im} \lambda \geq \alpha,|\lambda| \geq \delta$, and as $|\lambda| \rightarrow \infty$

$$
\hat{e}(x, \lambda)=e^{-i \lambda x}\left[1+O\left(\frac{1}{\lambda}\right)\right], \hat{e}^{\prime}(x, \lambda)=-i \lambda e^{-i \lambda x}\left[1+O\left(\frac{1}{\lambda}\right)\right]
$$

uniformly with respect to $x \geq 0$.

We denote

$$
f_{j}(x)=\frac{1}{2 \pi} \int_{-\infty}^{\infty}\left[\frac{\hat{\varphi}(\lambda)}{\varphi(\lambda)}-1\right] e^{i \lambda x} d \lambda=i \operatorname{Res}_{\lambda=\lambda_{j}} \frac{\hat{\varphi}(\lambda)}{\varphi(\lambda)} e^{i \lambda x},
$$

where $\left.\hat{\varphi}(\lambda)=\hat{e}^{\prime} 0, \lambda\right)-\left(\alpha_{0}+\alpha_{1} \lambda\right) \hat{e}(0, \lambda)$ and $j=1,2, \ldots, n$.

We shall call the polynomial

$$
P_{j}(x)=e^{-i \lambda_{j} x} f_{j}(x), \quad j=1,2, \ldots, n,
$$

with degree of $m_{j}-1$ the normalization polynomial for boundary value problem (1.1)-(1.3), where $m_{j}$ is the multiplicity of the singular number $\lambda_{j}(j=1,2, \ldots$, $n)$.

It turns out that the boundary value problem (1.1)-(1.3) is uniquely determined by the scattering function $S(\lambda)$, the non-real singular values $\lambda_{1}, \lambda_{2}, \ldots$, $\lambda_{n}$ and the normalization polynomials $P_{1}(x), P_{2}(x), \ldots, P_{n}(x)$. The set of values $\left\{S(\lambda), \lambda_{j}, P_{j}(x),(j=1,2, \ldots, n)\right\}$ is called the scattering data of the boundary value problem (1.1)-(1.3).

\section{Main equation}

Using the results in Section 2, we derive the main equation for boundary value problem (1.1)-(1.3) allowing the kernel $K(x, t)$ can be determined from the scattering data.

Theorem 3.1. For each fixed $x \geq 0$ the kernel $K(x, t)$ satisfies the following equation:

$$
F(x+\xi)+K(x, \xi)+\int_{x}^{\infty} K(x, t) F(t+\xi) d t=0, \quad x<\xi<\infty,
$$

where $F_{s}(x)$ and $F(x)$ will be defined by the formulas (3.4), (3.6), respectively.

Proof. Writing (1.4) in (2.1) we obtain

$$
\begin{aligned}
& 2 i \omega(x, \lambda)\left[\frac{\lambda}{\varphi(\lambda)}+\frac{1}{\alpha_{1}-i}\right]-\frac{2 i}{\alpha_{1}-i}\left[\omega(x, \lambda)-\left(\cos \lambda x+\alpha_{1} \sin \lambda x\right)\right] \\
= & {\left[S_{0}-S(\lambda)\right]\left\{e^{i \lambda x}+\int_{x}^{\infty} K(x, t) e^{i \lambda t} d t\right\} } \\
& +\int_{x}^{\infty} K(x, t) e^{-i \lambda t} d t+S_{0} \int_{-\infty}^{-x} K(x,-t) e^{-i \lambda t} d t .
\end{aligned}
$$


Let us multiply both sides of relation (3.2) by $\frac{1}{2 \pi} e^{i \lambda \xi}(\xi>x)$ and integrate this relation over $\lambda$ from $-\infty$ to $\infty$ :

$$
\begin{aligned}
& \frac{1}{2 \pi} \int_{-\infty}^{\infty} 2 i \omega(x, \lambda)\left[\frac{\lambda}{\varphi(\lambda)}+\frac{1}{\alpha_{1}-i}\right] e^{i \lambda \xi} d \lambda \\
& -\frac{1}{2 \pi} \int_{-\infty}^{\infty} \frac{2 i}{\alpha_{1}-i}\left[\omega(x, \lambda)-\left(\cos \lambda x+\alpha_{1} \sin \lambda x\right)\right] e^{i \lambda \xi} d \lambda \\
= & \frac{1}{2 \pi} \int_{-\infty}^{\infty}\left[S_{0}-S(\lambda)\right] e^{i \lambda(x+\xi)} d \lambda \\
& +\frac{1}{2 \pi} \int_{-\infty}^{\infty}\left[S_{0}-S(\lambda)\right]\left(\int_{x}^{\infty} K(x, t) e^{i \lambda(x+\xi)} d t\right) d \lambda \\
& +\frac{1}{2 \pi} \int_{-\infty}^{\infty}\left(\int_{x}^{\infty} K(x, t) e^{-i \lambda t} d t\right) d \lambda .
\end{aligned}
$$

On the right-hand side, taking $K(x, t)=0$ for $x>t$ into account, we obtain

$$
F_{s}(x+\xi)+K(x, \xi)+\int_{x}^{\infty} K(x, t) F_{s}(t+\xi) d t,
$$

where

$$
F_{s}(x)=\frac{1}{2 \pi} \int_{-\infty}^{\infty}\left[S_{0}-S(\lambda)\right] e^{i \lambda x} d \lambda .
$$

On the left-hand side of relation (3.3) using the Jordan's lemma and the residue theorem, we find that

$$
\begin{aligned}
& -i \sum_{\operatorname{Im} \lambda>0} \operatorname{Res}_{\lambda=\lambda_{i}} \frac{\hat{\varphi}(\lambda)}{\varphi(\lambda)} e^{i \lambda(x+\xi)}-i \int_{x}^{\infty} K(x, t) \sum_{\operatorname{Im} \lambda>0} \operatorname{Res}_{\lambda=\lambda_{i}} \frac{\hat{\varphi}(\lambda)}{\varphi(\lambda)} e^{i \lambda(t+\xi)} d t \\
= & -\sum_{j=1}^{n} f_{j}(x+\xi)-\int_{x}^{\infty} K(x, t) \sum_{j=1}^{n} f_{j}(t+\xi) d t
\end{aligned}
$$

where $f_{j}(x)$ is defined by $(2.12)$.

Thus, for $\xi>x$, taking the equalities (3.4) and (3.5) into account, from (3.3) we derive the relation

$$
\begin{aligned}
& -\sum_{j=1}^{n} f_{j}(x+\xi)-\int_{x}^{\infty} K(x, t) \sum_{j=1}^{n} f_{j}(t+\xi) d t \\
= & F_{s}(x+\xi)+K(x, \xi)+\int_{x}^{\infty} K(x, t) F_{s}(t+\xi) d t .
\end{aligned}
$$

We finally obtain

$$
F(x+\xi)+K(x, \xi)+\int_{x}^{\infty} K(x, t) F(t+\xi) d t=0, \quad x<\xi<\infty,
$$


where

$$
F(x)=\sum_{j=1}^{n} f_{j}(x)+F_{s}(x)
$$

The proof is completed.

The equation (3.1) is called the main equation or Gelfand-Levitan-Marchenko equation of the inverse problem of scattering theory for the boundary value problem (1.1)-(1.3).

Using the main equation (3.1), we obtain that the function $F(x)$ is differentiable on $[0, \infty)$ and its derivative satisfies the condition

$$
\int_{0}^{\infty}(1+x)\left|F^{\prime}(x)\right| d x<\infty
$$

and the function $S(\lambda)$ is continuous at all real points $\lambda$ (see [11, p. 210]).

Put

Then

$$
\tau(x)=\int_{0}^{\infty}\left|F^{\prime}(t)\right| d t, \quad \tau_{1}(x)=\int_{x}^{\infty} \tau(t) d t .
$$

$$
|F(x)| \leq \int_{x}^{\infty}\left|F^{\prime}(t)\right| d t=\tau(x), \quad \tau_{1}(0)<\infty
$$

holds.

\section{Uniqueness}

Lemma 4.1. Assume that the function $y_{x}(t)$ is summable on the $t \geq x$ half line and for $t \geq x$

$$
y_{x}(t)+\int_{x}^{\infty} y_{x}(u) F(u+t) d u=0 .
$$

Then $y_{x}(t) \equiv 0$ for $t \geq x$.

Proof. It suffices to prove that homogeneous equation (4.1) has only trivial solution $y_{x}(t) \equiv 0$ for $t \geq x$.

Let $z_{x}(t)$ be a solution of the integral equation

$$
y_{x}(t)=z_{x}(t)+\int_{x}^{t} K(\xi, t) z_{x}(\xi) d \xi
$$

for $t \geq x$, where the kernel $K(x, t)$ satisfies the equation (3.1) and (by (1.5)) the condition

$$
|K(\xi, t)| \leq c \sigma\left(\frac{\xi+t}{2}\right), \quad(c>0) .
$$

Then from (3.8) we have

$$
\int_{\xi}^{\infty}|F(t+u) K(\xi, u)| d u \leq c_{1} \tau_{1}(\xi)
$$


where $c_{1}>0$. Substituting (4.2) into (4.1) we obtain

$$
\begin{aligned}
z_{x}(t)+\int_{x}^{t} K(\xi, t) z_{x}(\xi) d \xi= & -\int_{x}^{\infty} z_{x}(\xi) F(\xi+t) d \xi \\
& -\int_{x}^{t} z_{x}(\xi) d \xi \int_{\xi}^{\infty} K(\xi, u) F(u+t) d u \\
& -\int_{t}^{\infty} z_{x}(\xi) d \xi \int_{\xi}^{\infty} K(\xi, u) F(u+t) d u .
\end{aligned}
$$

From the main equation (3.1) it's obtained

$$
\int_{\xi}^{\infty} K(\xi, u) F(t+u) d u=-[F(t+\xi)+K(\xi, t)]
$$

for $t \geq \xi$. Thus,

$$
z_{x}(t)=-\int_{t}^{\infty} z_{x}(\xi)\left[F(\xi+t)+\int_{\xi}^{\infty} K(\xi, u) F(u+t) d u\right] d \xi, \quad(t \geq \xi)
$$

holds. Hence, $z_{x}(t)$ is a solution of Volterra type homogenous integral equation for $t \geq x$. The kernel function of this integral equation is a rapidly decreasing function owing to (3.8) and (4.4). Therefore, we have $z_{x}(t) \equiv 0$ for $t \geq x$ and in view of (4.2) we have $y_{x}(t) \equiv 0$ for $t \geq x$. Lemma 4.1 is proved.

We obtain the following theorem from Lemma 4.1.

Theorem 4.2. The scattering data uniquely determine the boundary-value problem (1.1)-(1.3).

Proof. Obviously, to form main equation, it suffices to know the function $F(x)$. In turn, to find the function $F(x)$, it suffices to know only the scattering data of the boundary-value problem (1.1)-(1.3). Given the scattering data, we can use formulas $(3.4),(3.6)$ (also (2.13)) to construct the function $F(x)$ and write out the main equation (3.1) for the unknown function $K(x, t)$. It follows from Lemma 4.1 that the main equation has a unique solution. Solving this equation, we find the function $K(x, t)$, and by (1.6), hence potential $q(x)=-\frac{1}{2} \frac{d}{d x} K(x, x)$.

\section{References}

[1] T. Aktosun, Construction of the half-line potential from the Jost function, Inverse Problems 20 (2004), no. 3, 859-876.

[2] T. Aktosun and R. Weder, Inverse spectral-scattering problem with two sets of discrete spectra for the radial Schrödinger equation, Inverse Problems 22 (2006), no. 1, 89-114.

[3] C. T. Fulton, Singular eigenvalue problems with eigenvalue parameter contained in the boundary conditions, Proc. Roy. Soc. Edinburgh Sect. A 87 (1980/81), no. 1-2, 1-34.

[4] B. M. Levitan, On the solution of the inverse problem of quantum scattering theory, Mat. Zametki 17 (1975), no. 4, 611-624.

[5] Inverse Sturm-Liouville problems, Translated from the Russian by O. Efimov. VSP, Zeist, 1987. 
[6] V. E. Lyantse, An analog of the inverse problem of scattering theory for a non-selfadjoint operator, Mat. Sb. (N.S.) 72 (114) 1967 537-557.

[7] Kh. R. Mamedov, Uniqueness of the solution of the inverse problem of scattering theory for the Sturm-Liouville operator with a spectral parameter in the boundary condition, Mat. Zametki 74 (2003), no. 1, 142-146; translation in Math. Notes 74 (2003), no. 1-2, 136-140.

[8] Kh. R. Mamedov and H. Menken, On the inverse problem of scattering theory for a differential operator of the second order, Functional analysis and its applications, 185194, North-Holland Math. Stud., 197, Elsevier, Amsterdam, 2004.

[9] H. Menken H and Kh. R. Mamedov, On the inverse problem of the scattering theory for a boundary-value problem, Geometry, integrability and quantization, 226-236, Softex, Sofia, 2006

[10] V. A. Marchenko, On reconstruction of the potential energy from phases of the scattered waves, Dokl. Akad. Nauk SSSR (N.S.) 104 (1955), 695-698.

[11] Sturm-Liouville Operators and Applications, Translated from the Russian by A. Iacob. Operator Theory: Advances and Applications, 22. Birkhauser Verlag, Basel, 1986.

[12] M. A. Naimark, Linear Differential Operators. II, Frederick Ungar Publishing Co., New York, 1967.

[13] E. A. Počhěykina-Fedotova, The inverse boundary value problem on the half-axis for a second order equation, Izv. Vysš. Učebn. Zaved. Matematika 122 (1972), no. 7, 75-84.

[14] V. A. Yurko, On the reconstruction of the pencils of differential operators on the halfline, Mat. Zametki 67 (2000), no. 2, 316-320; translation in Math. Notes 67 (2000), no. $1-2,261-265$.

[15] _ An inverse problem for pencils of differential operators, Mat. Sb. 191 (2000), no. 10, 137-160; translation in Sb. Math. 191 (2000), no. 9-10, 1561-1586.

Department of Mathematics

Mersin University

33343 Mersin, Turkey

E-mail address: hanlar@mersin.edu.tr 